\section{Predicting Transformation to Type 2 Parenchymal Hematoma in Acute Ischemic Stroke by CT Permeability Imaging}

I read with great interest the retrospective study by Hom et al, ${ }^{1}$ in which a model combining clinical metrics with blood-brain barrier (BBB) permeability data from dynamic $\mathrm{CT}$ was presented to predict symptomatic hemorrhagic transformation (HT) and malignant edema in acute ischemic stroke. The authors stated that HT was classified according to the convention of the European Cooperative Acute Stroke Study (ECASS II), of which there are 4 subtypes $^{2}$ : hemorrhagic infarction types 1 and 2 (HI1 and HI2) and parenchymal hematoma types 1 and 2 ( $\mathrm{PH} 1$ and $\mathrm{PH} 2$ ). It was shown in ECASS II that PH2, defined as a space-occupying hematoma of $>30 \%$ of the infarct zone with substantial mass effect attributable to the hematoma, is the only subtype of HT that portends poor prognosis. ${ }^{3} \mathrm{HI}$ 's, on the other hand, are defined as petechial hemorrhages without mass effect and are likely epiphenomenal, reflecting reperfusion into the infarct. (For examples of the 4 HT subtypes by ECASS criteria, see Berger et al. ${ }^{3}$ )

The authors report that 3 of the 32 patients in their cohort (9.4\%) had HT that was classified as PH2, which is unusually high, especially given that not all patients received tissue plasminogen activator (tPA) (though the exact number of patients who did was not reported). $\mathrm{PH} 2$ is an uncommon event, occurring only in $1.7 \%$ of the 6444 intravenous tPA-treated patients in the Safe Implementation of Thrombolysis in Stroke-Monitoring Study ${ }^{4}$ and in $1.9 \%$ of the 418 intravenous tPA-treated patients in ECASS III. ${ }^{5}$ Presumably, the most illustrative of the $3 \mathrm{PH} 2 \mathrm{~s}$ was used by the authors for publication in Fig 1 (http:// www.ajnr.org/cgi/content/full/32/1/41/F1). However, looking at the follow-up CT at 23 hours postictus, the HT is petechial rather than a space-occupying hematoma, let alone one that would be categorized as PH2. While there is some mass effect and midline shift, these are to be expected, given the edema from the large infarct, and should not be attributed to the small focus of hemorrhage (red arrow in Fig 1). I believe the more appropriate classification of the HT shown in Fig 1 is HI2. Without strict adherence to the established ECASS classification scheme, the reader is left uncertain as to what the true incidence of $\mathrm{PH} 2$ was in this cohort and whether the technique of CT permeability imaging as described can actually predict the only sub-type of HT that is clinically relevant and most feared when administering thrombolytic or endovascular therapy.

Since our original report describing quantitative measurements of BBB permeability from dynamic CT perfusion imaging, ${ }^{6}$ several additional studies have appeared in the literature. ${ }^{7-10}$ Imaging protocols (ie, first-pass ${ }^{6,710}$ versus delayed acquisition ${ }^{8,9}$ ) and methods of modeling the dynamic data (Patlak model, ${ }^{8}$ modified delay-corrected Pat- lak model, ${ }^{6,10}$ distributed parameter model, ${ }^{7}$ adiabatic Johnson-Wilson model $^{9}$ ) vary considerably. A detailed comparison is beyond the space and scope of this brief letter, but a major concern that should be raised is the paradoxical depiction of nonzero permeability values in normal brain by Patlak modeling of delayed-acquisition data used in this study (again see Fig 1, as well as figures and tables from previous reports $^{8,10}$ ).

Clearly all of these methodologies require further validation. Despite the optimistic conclusions offered by Hom et al, ${ }^{1}$ I do not believe there are yet any compelling data that demonstrate the ability to predict $\mathrm{PH} 2$ unambiguously from other HT subtypes based on thresholds of permeability values derived from CT. As such, the use of dynamic CT permeability imaging for stroke triage is not warranted at this time, and the results of this article should not alter current clinical practice guidelines.

\section{References}

1. Hom J, Dankbaar JW, Soares BP, et al. Blood-brain barrier permeability assessed by perfusion CT predicts symptomatic hemorrhagic transformation and malignant edema in acute ischemic stroke. AJNR Am J Neuroradiol 2011;32:41-48

2. Larrue V, von Kummer RR, Muller A, et al. Risk factors for severe hemorrhagic transformation in ischemic stroke patients treated with recombinant tissue plasminogen activator: a secondary analysis of the European-Australasian Acute Stroke Study (ECASS II). Stroke 2001;32:438-41

3. Berger C, Fiorelli M, Steiner T, et al. Hemorrhagic transformation of ischemic brain tissue: asymptomatic or symptomatic? Stroke 2001;32:1330-35

4. Wahlgren N, Ahmed N, Dávalos A, et al. Thrombolysis with alteplase for acute ischaemic stroke in the Safe Implementation of Thrombolysis in Stroke-Monitoring Study (SITS-MOST): an observational study. Lancet 2007;369:275-82

5. Hacke W, Kaste M, Bluhmki E, et al. Thrombolysis with alteplase 3 to 4.5 hours after acute ischemic stroke. N Engl J Med 2008;359:1317-29

6. Lin K, Kazmi KS, Law M, et al. Measuring elevated microvascular permeability and predicting hemorrhagic transformation in acute ischemic stroke using first-pass dynamic perfusion CT imaging. AJNR Am J Neuroradiol 2007; 28:1292-98

7. Bisdas S, Hartel M, Cheong LH, et al. Prediction of subsequent hemorrhage in acute ischemic stroke using permeability CT imaging and a distributed parameter tracer kinetic model. J Neuroradiol 2007;34:101-08

8. Dankbaar JW, Hom J, Schneider T, et al. Dynamic perfusion CT assessment of the blood-brain barrier permeability: first pass versus delayed acquisition. AJNR Am J Neuroradiol 2008;29:1671-76

9. Aviv RI, d'Esterre CD, Murphy BD, et al. Hemorrhagic transformation of ischemic stroke: prediction with CT perfusion. Radiology 2009;250:867-77

10. Schneider T, Hom J, Bredno J, et al. Delay correction for the assessment of blood-brain barrier permeability using first-pass dynamic perfusion CT. AJNR Am J Neuroradiol 2010 Nov 24. [Epub ahead of print]

K. Lin

Department of Radiology

New York Hospital Medical Center of Queens

Flushing, New York

D0I 10.3174/ajnr.A2602 